\title{
Efficacy and safety of ambroxol hydrochloride in the treatment of secretory otitis media: a systematic review and meta-analysis
}

\author{
Xufeng Zhou", Xiulin Jin", Linhong Yang, Xiaoli Wei \\ Department of Otorhinolaryngology, The First Affiliated Hospital of Jiamusi University, Jiamusi, China \\ Contributions: (I) Conception and design: X Zhou; (II) Administrative support: None; (III) Provision of study materials or patients: X Jin; (IV) \\ Collection and assembly of data: L Yang; (V) Data analysis and interpretation: X Zhou; (VI) Manuscript writing: All authors; (VII) Final approval of \\ manuscript: All authors. \\ \#These authors contributed equally to this work. \\ Correspondence to: Xufeng Zhou. Department of Otorhinolaryngology, The First Affiliated Hospital of Jiamusi University, No. 348 Dexiang Street, \\ Xiangyang District, Jiamusi 154002, China. Email: zhouxufe2021@163.com.
}

\begin{abstract}
Background: Secretory otitis media is a very common nonsuppurative inflammatory disease in otorhinolaryngology. Ambroxol hydrochloride helps to improve ciliary movement in the ear canal and promote the dissolution and discharge of secretions. However, its effect still lacks systematic evaluation. We conducted a meta-analysis of clinical studies to systematically evaluate the application effect of ambroxol hydrochloride.

Methods: A computer-based search of the Chinese Biomedical Database (CBM), China National Knowledge Infrastructure (CNKI), PubMed, and Web of Science databases was conducted using the keywords "Ambroxol hydrochloride" \& "secretory otitis media". Randomized controlled trials published after 2015 were selected and then screened and analyzed using RevMan 5.4 software.

Results: Ten studies involving a total of 998 patients were included. Meta-analysis showed that adding ambroxol hydrochloride to the original glucocorticoid treatment improved therapeutic efficacy [odds ratio $(\mathrm{OR})=4.95,95 \%$ confidence interval $(\mathrm{CI}): 3.27,7.50, \mathrm{P}<0.00001]$, reduced tympanic pressure after treatment [mean difference $(\mathrm{MD})=-19.04,95 \% \mathrm{CI}:-22.72,-15.36, \mathrm{P}<0.00001]$, and increased the pure tone threshold $(\mathrm{MD}=6.37,95 \% \mathrm{CI}: 5.36,7.37, \mathrm{P}<0.00001)$, without increasing adverse reactions $(\mathrm{OR}=0.51,95 \% \mathrm{CI}: 0.14$, $1.85, \mathrm{P}=0.30)$.

Discussion: On the basis of the original treatment of secretory otitis media, adding ambroxol hydrochloride treatment improved the therapeutic effect, reduced tympanic pressure after treatment, and improved the pure tone threshold (hearing), without increasing adverse reactions.
\end{abstract}

Keywords: Ambroxol hydrochloride; secretory otitis media; meta-analysis

Submitted Nov 23, 2021. Accepted for publication Jan 18, 2022.

doi: $10.21037 / \mathrm{atm}-22-237$

View this article at: https://dx.doi.org/10.21037/atm-22-237

\section{Introduction}

Secretory otitis media is a nonsuppurative inflammatory disease of the middle ear that occurs in children and adults and is very common in otorhinolaryngology (1). The disease is mainly characterized by intratympanic effusion of the ear canal, combined with tinnitus, earache, ear swelling, and hearing loss symptoms, which negatively affect the quality of life of patients (2). At present, the pathogenesis of secretory otitis media is not clear and may be related to immune response, bacterial infection, eustachian tube dysfunction, gastroesophageal reflux, and other factors (3). Treatment approaches include surgical and nonsurgical methods. The main surgical methods include tympanocentesis, myringotomy, and tympanostomy tube insertion. Nonsurgical methods include antibiotics to 
control infection and glucocorticoids (dexamethasone or prednisone) to control inflammatory reactions (4). Ambroxol hydrochloride is a mucolytic agent and expectorant used in acute and chronic respiratory diseases to promote bronchial ciliary motility and reduce secretion viscosity (5). Recent studies have shown that the application of ambroxol hydrochloride in secretory otitis media can contribute to the dissolution of middle ear secretions, facilitate the exclusion of effusion and improvement of ciliary motor function, and contribute to the effects of inflammation, antioxidation, and mucosal protection (6). At present, there are no guidelines for the application of ambroxol hydrochloride in secretory otitis media, and there is a lack of systematic evaluation on the administration route, dosage, efficacy, and safety of this drug. Hou et al. (7) conducted a meta analysis on this topic in 2012, but this study didn't introduce subgroups and the quality of the included articles were not good enough. Our study included literatures with better quality and introduced subgroups according the administration methods of the drug, to provide better evidence.

We present the following article in accordance with the PRISMA reporting checklist (available at https://atm. amegroups.com/article/view/10.21037/atm-22-237/rc).

\section{Methods}

\section{Inclusion criteria}

(I) Study type: randomized controlled trials (RCTs) limited to the past 5 years (literature published after January 2015) and published in English or Chinese. (II) Study subjects: all patients who were diagnosed with secretory otitis media and had secretory fluid withdrawn during tympanostomy, regardless of patient age and disease in one or both ears, and excluding otitis media caused by spaceoccupying lesions of the nasal cavity or nasopharyngitis. (III) Intervention methods: studies that included an observation and control group, with patients in both groups receiving basic treatment methods, including routine disinfection, topical local anesthesia of the periosteum and external auditory canal, puncture of the tympanic membrane with a syringe, back effusion, intratympanic drug injection (dexamethasone), and for the observation group, additional ambroxol hydrochloride treatment (oral or intratympanic injection) was added. (IV) Efficacy determination: studies that included recovery criteria (the patient's hearing returned to normal, no tinnitus, and no ear plug symptoms), effective treatment criteria (the patient's hearing recovered and symptoms abated), ineffective treatment criteria (patient had no abatement of symptoms, no change, or worsening of hearing), and response rate $=$ (recovered cases + effective cases)/total cases.

\section{Literature search strategy}

We conducted a computer-based search of the Chinese Biomedical Database (CBM), China National Knowledge Infrastructure (CNKI), PubMed, and Web of Science databases. The search method was keyword rapid search and the search terms were "Ambroxol hydrochloride" \& "secretory otitis media". We only selected RCTs published after 2015.

\section{Literature selection and data extraction}

Two researchers independently screened the literature. If there was inconsistency in this process, a $3 \mathrm{rd}$ person was required to reach an agreement through negotiation. Excel was used for data extraction, including: (I) basic data of the literature (publication time, author, and region), (II) characteristics of the study subjects (patient age, gender, and air conduction threshold), (III) intervention methods and treatment time of the 2 groups, and (IV) outcome data. If there were data not provided in the literature, they were obtained according to the address specified in the literature. If the data could not be obtained, the original author of the literature was contacted to obtain the data; if the data could not be obtained from the author, the study was excluded.

\section{Outcome indicators}

Efficacy indicators were: (I) treatment response rate, (II) tympanic pressure, (III) hearing [measured by pure tone threshold (db)] and (IV) adverse reactions.

Indicators for which no statistical analysis was done included: serum high-sensitivity C-reactive protein (hsCRP), interleukin (IL)-2, IL-6, and IL-8 levels after treatment; recurrence rate; and quality of life after treatment.

The reason for not performing statistical analysis on the above indicators was because: (I) the number of studies reporting some indicators was too small (for example, only 2 studies reported recurrence rate dosage; and (II) reporting on indicators was not uniform among the different studies (for example, the evaluation tables adopted for assessing quality of life varied). 


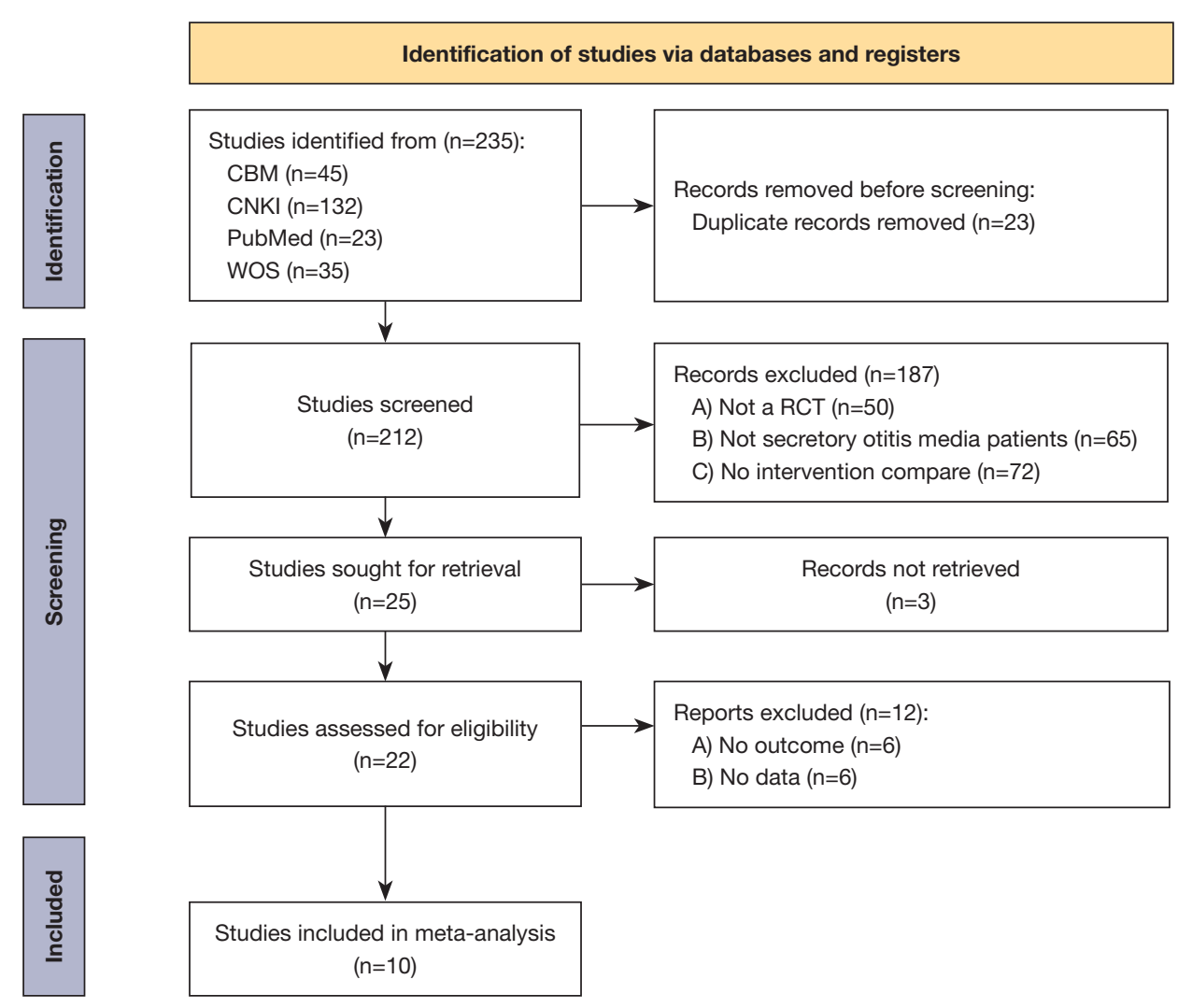

Figure 1 The selection flow chart.

\section{Statistical methods}

Heterogeneity between the different studies was determined using $\mathrm{I}^{2}$ test analysis and $Q$ test. $\mathrm{I}^{2}<50 \%$ or $\mathrm{P} \geq 0.1$ indicated no statistical significance of heterogeneity, indicating that there was no or acceptable heterogeneity between the studies. Mean difference (MD) effect size was used for continuous variables, odds ratio (OR) effect size was used for binary variables, $95 \%$ was used as the confidence interval $(\mathrm{CI})$, and $\mathrm{P}<0.05$ was considered statistically significant. For each outcome indicator, all studies reporting the indicator were combined for statistical analysis. If there was no statistical heterogeneity among the studies, a fixed-effect model was used. If there was heterogeneity, a random-effects model was used. RevMan 5.4 software provided by Cochrane was used to present the results of analysis in the form of a forest plot, and publication bias was reported in a funnel plot.

\section{Risk of bias, heterogeneity survey, and sensitivity analysis}

The risk of bias assessment tool in RevMan 5.4 was used for analysis. Risk assessment was performed for each study on
6 aspects: randomization, allocation concealment, quality of blinding, outcome assessment, incomplete data, selective reporting, and other bias. The quality of the studies was assessed using the Jadad scale, and a score of more than 3 was considered a high-quality study. If there was heterogeneity in the statistical process, the source of heterogeneity was judged by subgroup analysis. If the source could not be confirmed, general descriptive analysis was used. Sensitivity analysis was performed by comparing the results of the fixed-effect model with those of the random-effects model.

\section{Results}

\section{Literature screening results}

In our search, 235 documents were initially identified, and 10 studies involving a total of 998 patients were finally included in the meta-analysis (Figure 1).

\section{Basic characteristics of the literature}

Basic characteristics of the literature (8-17) are shown in 
Table 1 . The youngest study subject was 11 years old and the oldest was 77 years old. The intervention method of 1 study (11) for the observation group was ambroxol hydrochloride without glucocorticoid (dexamethasone), while the administration route of 4 studies $(12,13,16,17)$ was oral. The administration route of the other studies was intratympanic injection. The shortest treatment time of the 8 studies was 1 week, and the longest was 4 weeks.

\section{Risk assessment of bias of included literature}

RevMan 5.4 was used for the risk assessment of the included studies. One study (8) only divided the patients into observation group and control group according to treatment method, and as random allocation was not used, there was the possibility of selection bias. None of the studies described the blind method or allocation concealment, potentially leading to implementation risk. None of the studies described dropout cases in detail, which may have led to incomplete data. There was sign of selective reporting or other bias risks (Figures 2,3).

\section{Meta analysis results}

\section{Clinical response rate}

All included studies (8-17) reported the efficacy of ambroxol hydrochloride in the treatment of secretory otitis media. There was no statistical heterogeneity between studies $\left(\mathrm{I}^{2}=0 \%, \mathrm{P}=0.82\right)$, and therefore, the fixed-effect model was used for combined analysis. The effectiveness rate of ambroxol hydrochloride in the observation group was higher than that in the control group ( $\mathrm{OR}=4.95,95 \% \mathrm{CI}$ : $3.27,7.50, \mathrm{P}<0.00001)$.

The studies were further divided into 2 subgroups according to the route of administration: intratympanic injection and oral administration. There was no statistical heterogeneity in the 2 groups. The efficacy of the 2 groups was higher than that of the control group (Figure 4).

\section{Posttreatment tympanic pressure}

Four studies $(8,9,11,14)$ reported tympanic pressure after treatment, with 186 patients in both the experimental group and the control group. There was statistical heterogeneity between the articles $\left(\mathrm{I}^{2}=77 \%, \mathrm{P}=0.005\right)$, and thus a randomeffects model was used for combined analysis. The results showed that treatment with ambroxol hydrochloride reduced tympanic pressure after treatment $(M D=-19.04$, 95\% CI: $-22.72,-15.36, \mathrm{P}<0.00001$; Figure 5).

\section{Auditory threshold (db)}

Six studies $(8,9,11,14-16)$ reported the pure tone threshold of patients after treatment. There was statistical heterogeneity between the studies $\left(\mathrm{I}^{2}=60 \%, \mathrm{P}=0.03\right)$, and thus the random-effect model was used for combined analysis. The results showed that the pure tone threshold of patients after treatment with ambroxol hydrochloride was higher than that of the control group ( $\mathrm{MD}=6.37,95 \% \mathrm{CI}$ : 5.36, 7.37, $\mathrm{P}<0.00001$; Figure 6).

\section{Adverse reactions}

There were 4 studies $(8,11,12,15)$ that reported adverse reactions after ambroxol hydrochloride treatment. As there was statistical heterogeneity between the studies $\left(\mathrm{I}^{2}=54 \%\right.$, $\mathrm{P}=0.09$ ), the random-effects model was used for combined analysis. There was no significant difference in adverse reactions between the ambroxol hydrochloride treatment group and the control group (OR $=0.51,95 \%$ CI: $0.14,1.85$, $\mathrm{P}=0.30$; Figure 7).

\section{Heterogeneity investigation and sensitivity analysis}

There was no statistically significant heterogeneity among the studies for treatment response rate indicators. Further, the literature was analyzed according to the administration route. However, there was still no heterogeneity among the studies in the subgroup. If the random-effects model was used for analysis, the results were not significantly different from those of the fixed-effect model. Sensitivity analysis thus showed that the results could be considered stable.

\section{Analysis of publication bias}

The funnel plot of ambroxol hydrochloride in the treatment of secretory otitis media showed that both sides were evenly distributed, suggesting that there was no publication bias (Figure 8).

\section{Discussion}

Ambroxol hydrochloride was originally used as an expectorant to enhance the ciliary motility of the respiratory system and is often used in the treatment of respiratory diseases (18). Guidelines and systematic reviews are lacking for the treatment of secretory otitis media, which is not supported by scientific evidence-based research. Eight RCT studies were included in this study. On the basis of the original glucocorticoid treatment, the addition of ambroxol hydrochloride improved the therapeutic effect, reduced tympanic pressure after treatment, and increased 


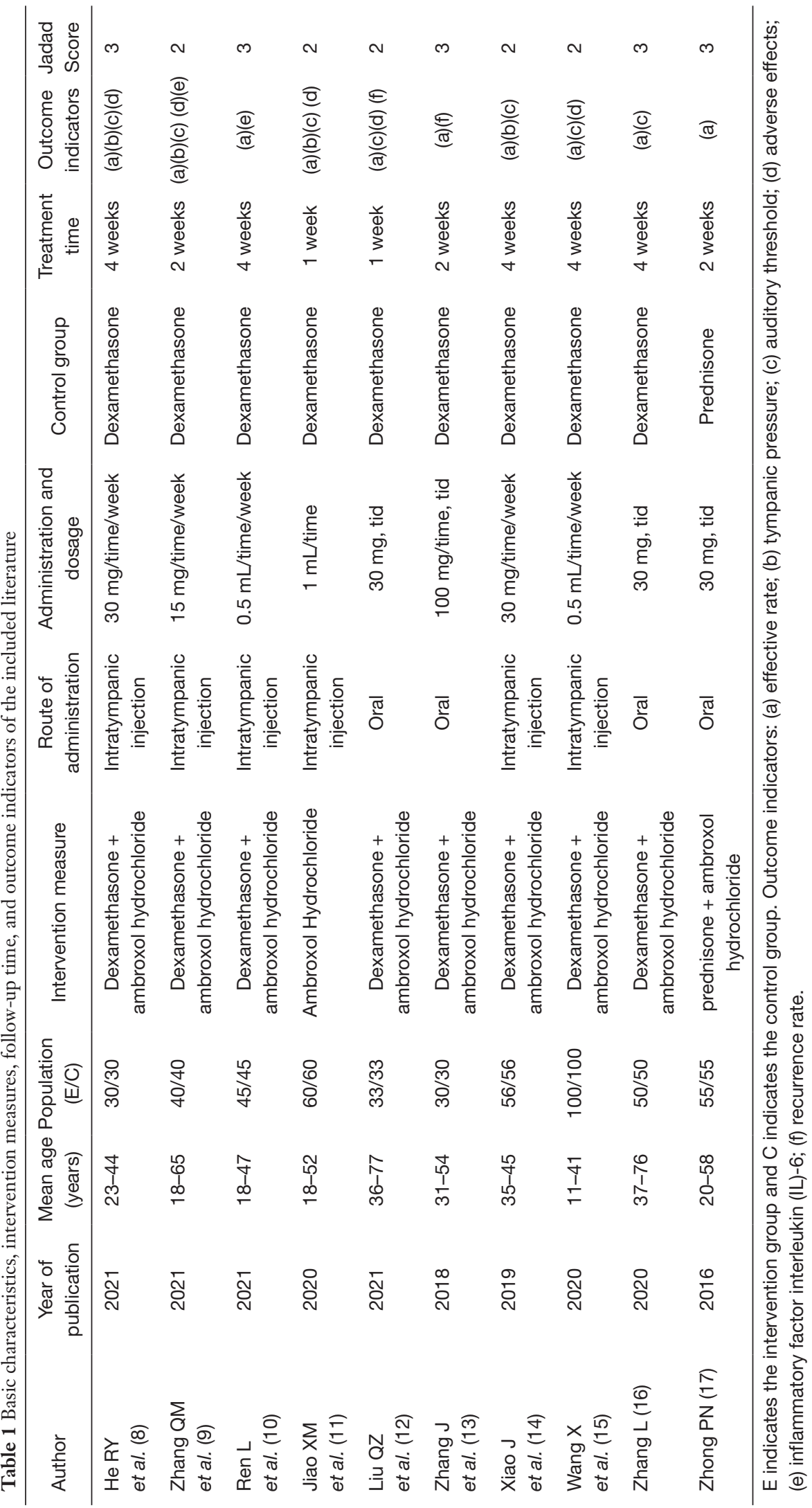


the pure tone threshold (hearing), without increasing adverse reactions. Consistent with the principle of treating respiratory diseases, ambroxol hydrochloride can adjust the ratio of gel layer to stained gel layer in the mucociliary system of the middle ear canal and increase the ciliary

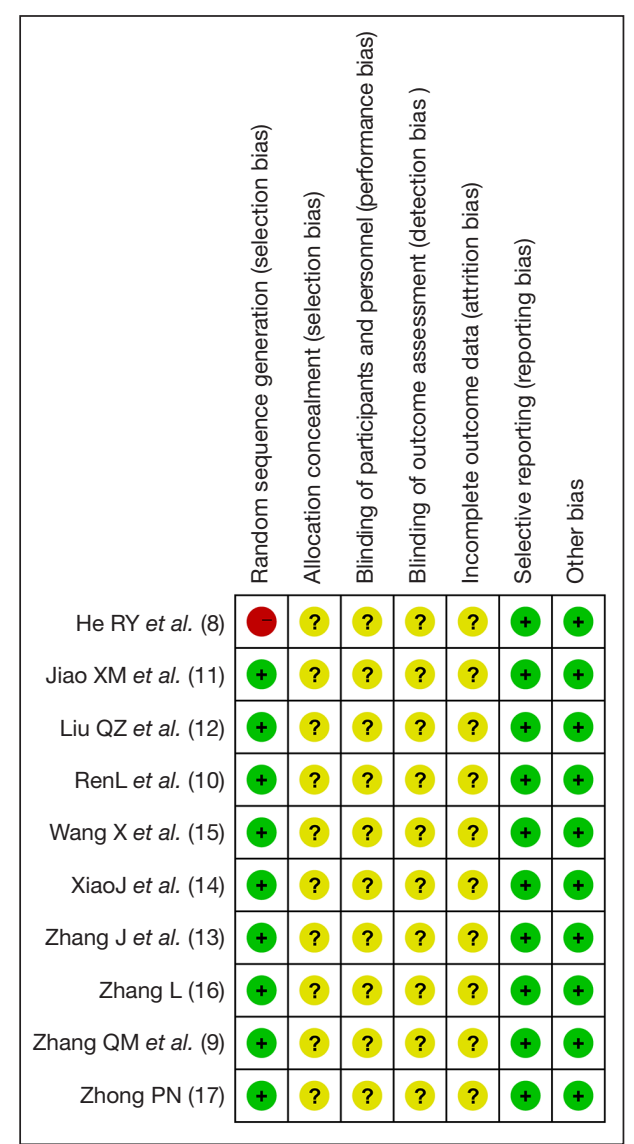

Figure 2 Summary chart of risk of bias assessment of included literature. transport function, thus facilitating the discharge of effusion (19). In addition, ambroxol hydrochloride can be used as a surfactant in the eustachian tube and middle ear mucosa, destroying free radicals and thus promoting the dilution and dissolution of effusion (20).

One study (9) measured and compared inflammatory factors in the secretions of patients before and after treatment. The results showed that after treatment for 14 days, the expression of inflammatory factors IL-6, IL-8, and tumor necrosis factor $\alpha(\mathrm{TNF}-\alpha)$ in the secretions of the observation group was lower than those of the control group, suggesting that ambroxol hydrochloride was beneficial for reducing the inflammatory response. The reason for this may be related to the discharge of secretions, the normalization of ciliary function, and the benefit to the control of local inflammation. Ambroxol hydrochloride can regulate the characteristics of secretions, effectively inhibit the release of inflammatory mediators, and promote the bactericidal and bacteriostatic effects of antibacterial drugs (21). The $\mathrm{T}$ cell subgroup parameters $\mathrm{CD}^{+}$and $\mathrm{CD} 8^{+}$of secretory otitis media can be used as another sensitive reference index for the curative effect. When the middle ear is attacked by pathogenic factors, the body will automatically generate immune defense, resulting in the disorder of $\mathrm{CD}^{+}$and $\mathrm{CD}^{+}$cell levels and aggravating the patient's condition. Ambroxol hydrochloride can significantly improve the patient's immune function and restore the immune cell indexes to the normal level, so as to improve the anti-inflammatory ability of patients (22).

The incidence of secretory otitis media is related to infection, immunity and eustachian tube disorder, Glucocorticoids (represented by dexamethasone) can inhibit the division and proliferation of macrophages, inhibit the reaction of tympanic mucosal cells, and further reduce the synthesis and release of inflammatory mediators, showing

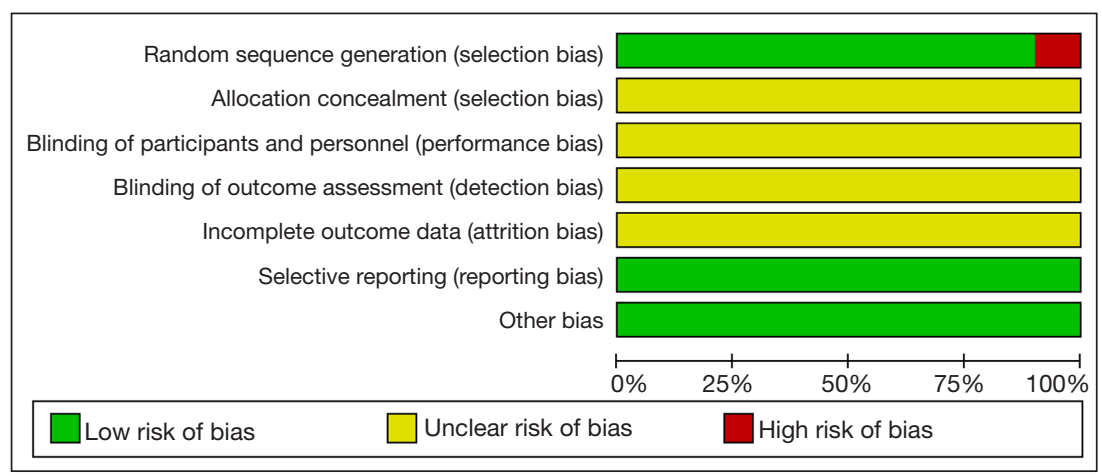

Figure 3 Risk of bias assessment chart of the included literature. 


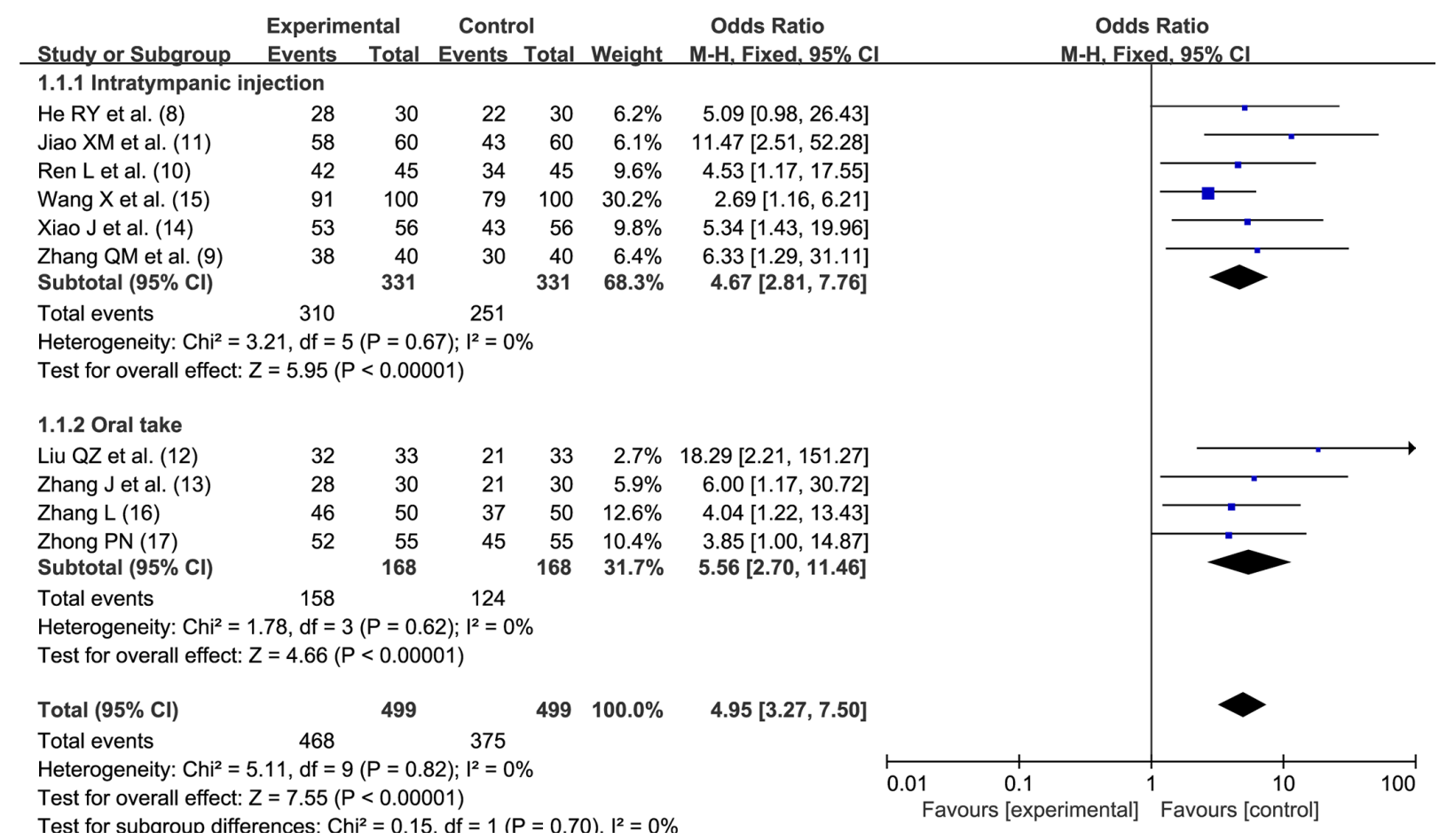

Figure 4 Efficacy of ambroxol hydrochloride in the treatment of secretory otitis media. M-H, Mantel-Haenszel; CI, confidence interval.

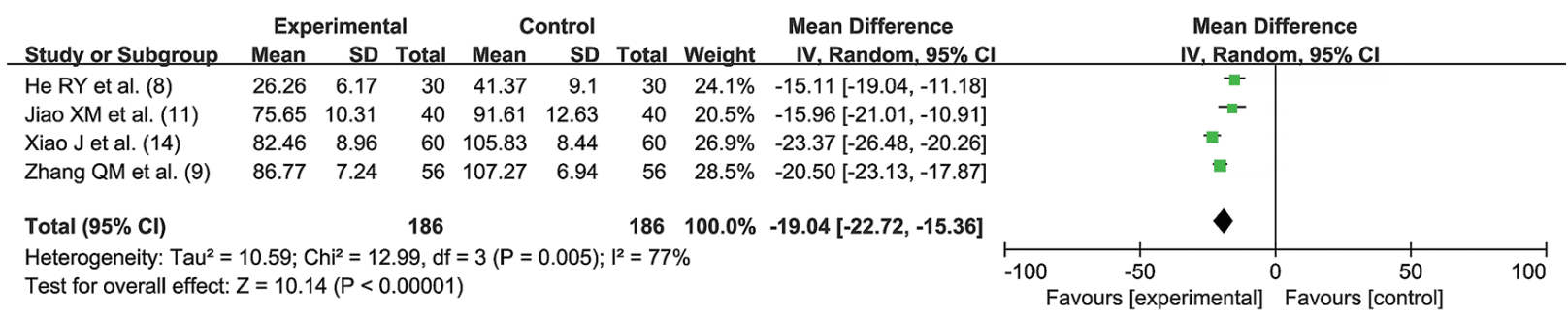

Figure 5 Effect of ambroxol hydrochloride on tympanic pressure after treatment of secretory otitis media. SD, standard deviation; CI, confidence interval; IV, inverse variance.

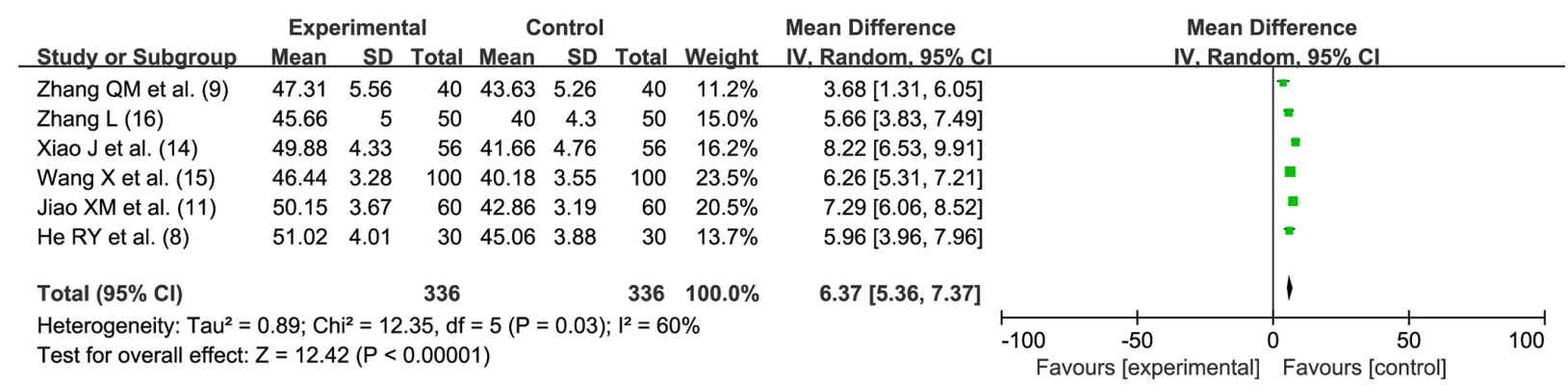

Figure 6 Effect of ambroxol hydrochloride on pure tone threshold after treatment of secretory otitis media. SD, standard deviation; CI, confidence interval; IV, inverse variance. 


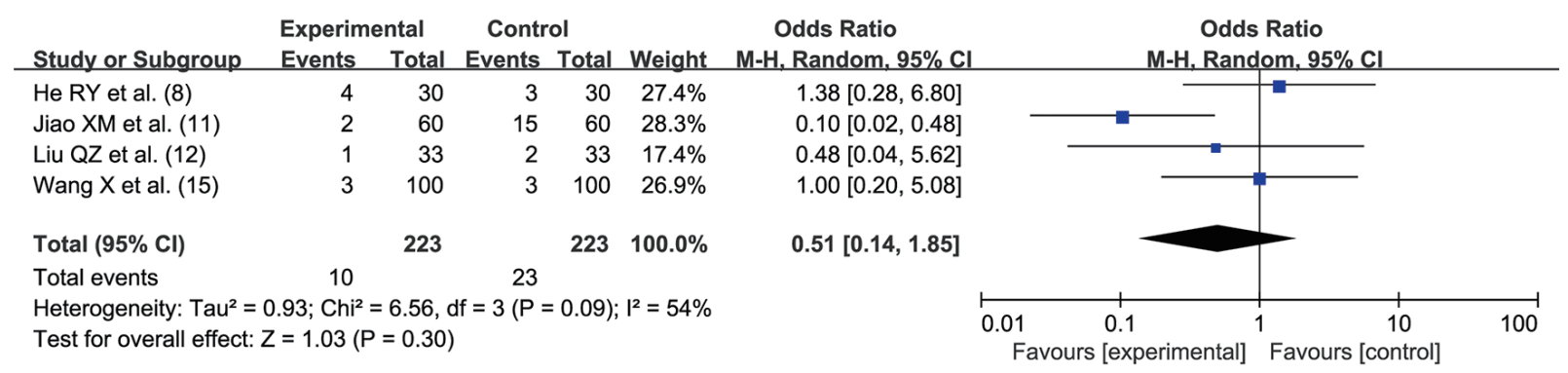

Figure 7 Adverse reactions of ambroxol hydrochloride in the treatment of secretory otitis media. M-H, Mantel-Haenszel; CI, confidence interval.

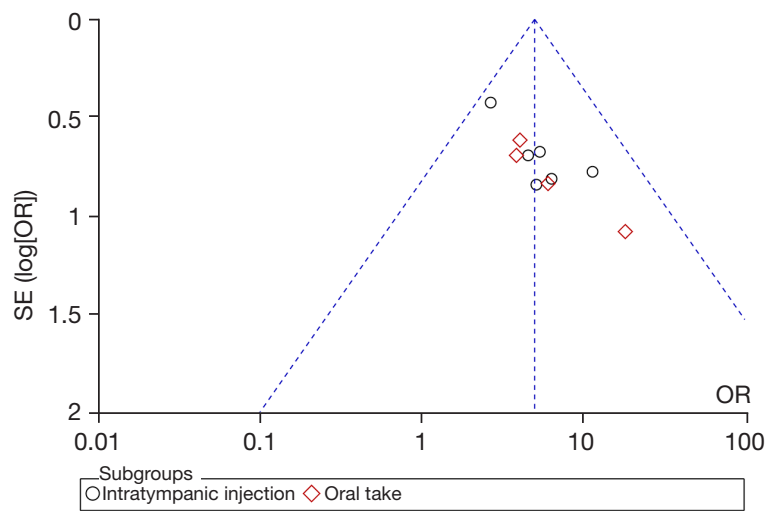

Figure 8 Funnel plot of ambroxol hydrochloride in the treatment of secretory otitis media. OR, odd ratio; SE, standard error.

a strong anti-inflammatory effect; ambroxol hydrochloride has a good effect of dissolving mucus, which can effectively reduce the viscosity of middle ear secretion, promote its dissolution, and accelerate the discharge of middle ear effusion, glucocorticoids play a better therapeutic role together with ambroxol hydrochloride (6). In the study of Liu et al. (12), the control group was treated with ambroxol hydrochloride alone, and the observation group was treated with dexamethasone combined with ambroxol hydrochloride, the results showed that the curative effect of the observation group was higher than that of the control group. The study pointed out that the single application of ambroxol hydrochloride in the treatment of secretory otitis media is prone to complications and lead to repeated diseases, which will hinder the patient's physical recovery, but combined with glucocorticoid treatment, it can gradually reduce the eustachian tube mucosal edema while anti-allergy and anti-inflammatory.

Adverse reactions after adding ambroxol hydrochloride were reported in 4 of the studies $(8,11,12,15)$, including nausea, vomiting, rash, elevated transaminases, and elevated blood glucose, but there was no significant difference compared with the control group.

In this study, the literature was divided on the basis of administration route into intratympanic injection and oral administration. The effective rate of intratympanic injection was $\mathrm{OR}=4.67,95 \%$ CI: 2.81, 7.76, while the effective rate of oral administration was $\mathrm{OR}=5.56,95 \% \mathrm{CI}: 2.70$, 11.46. The effective rate of oral ambroxol hydrochloride was higher than that of intratympanic injection. However, oral administration was reported in only 4 of the included studies, and therefore the difference in efficacy between the 2 administration methods still needs to be proven by a randomized controlled study. A study by Okunlola (23) have suggested that intratympanic injection acts directly on the site of middle ear lesions compared with oral administration, which is more conducive to effusion discharge, improving ear ventilation function and prognosis. In another study by $\mathrm{Li}$ (24) ambroxol hydrochloride was administrated by eustachian tube guided under nasal endoscope, the result showed more effective than intratympanic injection, which could be another new topic for the future study.

In this study, the literature was internally homogeneous in the meta-analysis of treatment effectiveness. The funnel plot showed that both sides were evenly distributed without significant publication bias. Sensitivity analysis showed that the results were stable. However, our study included only 10 studies, the quality of the studies was relatively low, some studies did not use the random allocation, and none described the dropout cases in detail, possibly leading to bias. Therefore, further evidence from a larger sample and higher quality RCTs is needed.

\section{Conclusions}

Ten studies were included in this meta-analysis, which 
showed that for secretory otitis media, adding ambroxol hydrochloride treatment on the basis of the original treatment improved therapeutic effect, reduced the tympanic pressure after treatment, and improved the pure tone threshold (hearing), without increasing adverse reactions.

\section{Acknowledgments}

Funding: None.

\section{Footnote}

Reporting Checklist: The authors have completed the PRISMA reporting checklist. Available at https://atm. amegroups.com/article/view/10.21037/atm-22-237/rc

Conflicts of Interest: All authors have completed the ICMJE uniform disclosure form (available at https://atm. amegroups.com/article/view/10.21037/atm-22-237/coif). The authors have no conflicts of interest to declare.

Ethical Statement: The authors are accountable for all aspects of the work in ensuring that questions related to the accuracy or integrity of any part of the work are appropriately investigated and resolved.

Open Access Statement: This is an Open Access article distributed in accordance with the Creative Commons Attribution-NonCommercial-NoDerivs 4.0 International License (CC BY-NC-ND 4.0), which permits the noncommercial replication and distribution of the article with the strict proviso that no changes or edits are made and the original work is properly cited (including links to both the formal publication through the relevant DOI and the license). See: https://creativecommons.org/licenses/by-nc-nd/4.0/.

\section{References}

1. Harmes KM, Blackwood RA, Burrows HL, et al. Otitis media: diagnosis and treatment. Am Fam Physician 2013;88:435-40. Erratum in: Am Fam Physician 2014;89:318. Dosage error in article text.

2. Venekamp RP, Damoiseaux RA, Schilder AG. Acute Otitis Media in Children. Am Fam Physician 2017;95:109-10.

3. Shirai N, Preciado D. Otitis media: what is new? Curr Opin Otolaryngol Head Neck Surg 2019;27:495-8.

4. Bhutta MF, Thornton RB, Kirkham LS, et al.
Understanding the aetiology and resolution of chronic otitis media from animal and human studies. Dis Model Mech 2017;10:1289-300.

5. Liu H, Wang W, Gao X. Comparison of the efficacy of ambroxol hydrochloride and $\mathrm{N}$-acetylcysteine in the treatment of children with bronchopneumonia and their influence on prognosis. Exp Ther Med 2020;20:130.

6. Passàli D, Zavattini G. Multicenter study on the treatment of secretory otitis media with ambroxol. Importance of a surface-tension-lowering substance. Respiration 1987;51 Suppl 1:52-9.

7. Hou S, Yang HZ. Meta analysis of ambroxol hydrochloride in the treatment of secretory otitis media. Journal of Shandong University 2012;26:10-2.

8. He RY, Huang ZW. Clinical study of intratympanic injection of dexamethasone and ambroxol in the treatment of secretory otitis media. Shenzhen Journal of Traditional Integrated Chinese and Western Medicine 2021;31:32-4.

9. Zhang QM, Mao Z, Huo YT, et al. Effect of intratympanic injection of ambroxol combined with dexamethasone on secretory otitis media and expression of inflammatory factors in secretions. Chinese Medical Innovation 2021;18:35-9.

10. Ren L, Li L. Clinical effect of intratympanic injection of dexamethasone combined with ambroxol in the treatment of secretory otitis media and its effect on the levels of IL2, IL-6, LTD4 and vascular permeability. Clinical Medical Research and Practice 2021;6:103-5.

11. Jiao XM. Clinical effect of tympanic injection of ambroxol hydrochloride in the treatment of secretory otitis media. Chinese Contemporary Medicine 2020;27:160-2.

12. Liu QZ. Clinical efficacy and safety of glucocorticoid combined with ambroxol hydrochloride in the treatment of secretory otitis media. Journal of Clinical Rational Drug Use 2021;14:104-6.

13. Zhang J. Effect analysis of glucocorticoid combined with ambroxol hydrochloride in the treatment of secretory otitis media. Systems Medicine 2018;3:31-3.

14. Xiao J, Wang Y, Tu EY. Efficacy of ambroxol hydrochloride intraventricular injection combined with Pulmicort Respules atomization in the treatment of secretory otitis media and its effect on patients' ear effusion and serum PCT and hs CRP. Hainan Medical Journal 2019;30:2106-9.

15. Wang X. Clinical efficacy analysis of ambroxol hydrochloride combined with dexamethasone in the treatment of secretory otitis media. Clinical Research 2020;28:48-9. 


\section{Page 10 of 10}

16. Zhang L. Observation on the effect of glucocorticoid combined with ambroxol hydrochloride in the treatment of secretory otitis media. Contemporary Medicine 2020;26:30-2.

17. Zhong PN. Effect of ambroxol hydrochloride on secretory otitis media. Northern Pharmacy 2016;13:76-7.

18. Olaleye OA, Kaur M, Onyenaka CC. Ambroxol Hydrochloride Inhibits the Interaction Between Severe Acute Respiratory Syndrome Coronavirus 2 Spike Protein 's Receptor Binding Domain and Recombinant Human ACE2. Preprint. bioRxiv. 2020; 2020.09.13.295691.

19. Sartoris A. Treatment of secretory otitis media with ambroxol. Acta Otorhinolaryngol Ital 1987;7 Suppl 16:51-60.

20. Calero del Castillo JB, Pérez Martínez F, Piqueras Pérez $\mathrm{F}$, et al. Incorporation of a stimulant of the synthesis and secretion of pulmonary surfactant (ambroxol) in the medical treatment of serous stimulotitis. An

Cite this article as: Zhou X, Jin X, Yang L, Wei X. Efficacy and safety of ambroxol hydrochloride in the treatment of secretory otitis media: a systematic review and meta-analysis. Ann Transl Med 2022;10(3):142. doi: 10.21037/atm-22-237

\section{Zhou et al. Ambroxol hydrochloride for secretory otitis media}

Otorrinolaringol Ibero Am 1988;15:209-18.

21. Bouscary A, Quessada C, Mosbach A, et al. Ambroxol Hydrochloride Improves Motor Functions and Extends Survival in a Mouse Model of Familial Amyotrophic Lateral Sclerosis. Front Pharmacol 2019;10:883.

22. Xiaorong D, Li M, Xuefeng L, et al. Effects of dexamethasone combined with ambroxol hydrochloride on T-Cell subsets and hearing in patients with secretory otitis media. Pak J Pharm Sci 2019;32:1437-40.

23. Okunlola A, Odeniyi MA, Arhewoh MI. Microsphere formulations of ambroxol hydrochloride: influence of Okra (Abelmoschus esculentus) mucilage as a sustained release polymer. Prog Biomater 2020;9:65-80.

24. Li XL, He ZX, Zhang ZF. Comparative study on the efficacy of ambroxol hydrochloride in the treatment of secretory otitis media. Traffic Medicine 2019;33:188$189+195$. 